\title{
Ultrasound and microbubble induced release from intracellular compartments
}

Farah Hussein ${ }^{1}$, Costin Antonescu, ${ }^{2,4}$ and Raffi Karshafian ${ }^{1,3,4^{*}}$ (D)

\begin{abstract}
Background: Ultrasound and microbubbles (USMB) have been shown to enhance the intracellular uptake of molecules, generally thought to occur as a result of sonoporation. The underlying mechanism associated with USMB-enhanced intracellular uptake such as membrane disruption and endocytosis may also be associated with USMB-induced release of cellular materials to the extracellular milieu. This study investigates USMB effects on the molecular release from cells through membrane-disruption and exocytosis.

Results: USMB induced the release of $19 \%$ and $67 \%$ of GFP from the cytoplasm in viable and non-viable cells, respectively. Tfn release from early/recycling endosomes increased by $23 \%$ in viable cells upon USMB treatment. In addition, the MFI of LAMP-1 antibody increased by $50 \%$ in viable cells, suggesting USMB-stimulated lysosome exocytosis. In non-viable cells, labeling of LAMP-1 intracellular structures in the absence of cell permeabilization by detergents suggests that USMB-induced cell death correlates with lysosomal permeabilization.

Conclusions: In conclusion, USMB enhanced the molecular release from the cytoplasm, lysosomes, and early/ recycling endosomes.

Keywords: Ultrasound and microbubble, Sonoporation, Endocytosis, Exocytosis, Intracellular uptake, Intracellular release, Acoustic cavitation bioeffects, In vitro ultrasound bioeffects, Cellular bioeffects of ultrasound
\end{abstract}

\section{Background}

The efficiency of drug therapy partly depends on the amount of drug delivered to the target cell and the release of the drug from the cell [1]. Uptake and release of molecules in cells can occur through intrinsic cellular processes such as endocytosis and exocytosis, respectively $[2,3]$, as well as through intrinsic membrane transporters for specific molecules such as glucose or ions [2]. The intracellular delivery of macromolecules can be enhanced through the application of ultrasound and microbubbles (USMB) by inducing plasma membrane disruption [4-7] and enhancing endocytosis [8-10]. In addition, USMB can also induce the release of cytoplasmic content and exocytosis of lysosomes immediately following exposure [11-13]. However, the bioeffects on the lysosomes that remain inside the cell without fusing

\footnotetext{
* Correspondence: karshafian@ryerson.ca

'Department of Physics, Ryerson University, 350 Victoria Street Toronto, Ontario M5B 2K3, Canada

${ }^{3}$ Institute for Biomedical Engineering, Science and Technology (iBEST),

Toronto, Canada

Full list of author information is available at the end of the article
}

with the plasma membrane and the release from other membrane bound cellular compartments are still unknown. In addition, the effect of time on the cellular release of molecules following USMB is not known.

Studies have shown that USMB can enhance the intracellular uptake of molecules, which otherwise would be excluded from cells, through mechanically inducing porelike plasma membrane disruption in a phenomenon known as sonoporation $[4,5,14]$. In addition, USMB enhances the intracellular uptake of molecules through endocytosis, which is an active mechanism of molecular uptake in biological cells [8]. Molecules that enter a cell through membrane disruptions are localized in the cytoplasm, while molecules that enter through endocytosis are initially localized within membrane bounded vesicles $[8,10]$. These vesicles can be transported to recycling endosomes, where some proteins and receptors are recycled back to the cell surface or subjected to membrane traffic to lysosomes for degradation [15]. The USMB enhanced endocytosis may be associated with the mechanism of membrane repair, perhaps resulting in internalization of the damaged part of the plasma membrane, as is 
known to occur in response to bacterial pore-forming toxins [16]. The dominant mechanism of enhanced uptake may be partly dependent on the size of the delivered molecules [8].

USMB may induce the release of cellular contents into the extracellular space by diffusion through disruptions on the plasma membrane, as shown by the release of hemoglobin from human erythrocytes [12] and Green Fluorescent Protein (GFP) from GFP-transfected HeLa cells [11] immediately following exposure to USMB. However, the release of the cytosolic indicator in these studies did not differentiate between viable and nonviable cells following exposure. As non-viable cells are expected to exhibit disruption of membrane integrity, distinguishing the release of material from viable $v s$. non-viable cells is an important consideration. In addition, USMB can induce lysosomal exocytosis immediately following exposure, triggered by the influx of $\mathrm{Ca}^{2+}$ ions through the membrane disruptions [13]. However, the broader bioeffects of USMB on endomembrane traffic, including the effects on recycling or plasma membrane fusion of intracellular lysosomes and early/ recycling endosomes, thus controlling release of their contents is not well understood. This study examines the effects of USMB on the release of molecules from specific cell compartments, including the cytoplasm, lysosomes, and early/recycling endosomes up to $21.5 \mathrm{~min}$ following exposure. The hypothesis guiding this study is that USMB can enhance the release of molecules from cells through membrane disruption and enhanced exocytosis. Experiments were conducted in vitro using Retinal Pigmented Epithelial (RPE) cells that were treated with USMB and analyzed using flow cytometry and microscopy. The results obtained from this study have demonstrated that USMB enhanced the release of molecules from cells.

\section{Methods}

The effects of USMB on the release of molecules from early/recycling endosomes, cytoplasm, and lysosomes at $1.5 \mathrm{~min}, 11.5 \mathrm{~min}$, and $21.5 \mathrm{~min}$ following the start of USMB were examined. This was done by tracking either Alexa647-Transferrin, GFP signal in RPE cells stably transfected with a soluble GFP-tagged protein (clathrin), and lysosomal associated membrane protein-1 (LAMP-1) using specific antibodies, respectively [17, 18]. Notably, soluble transferrin is a well-established marker of the early and recycling endosomes, as is LAMP-1 of the late endosome/lysosomes and these two markers are mostly non-overlapping in diverse types of cells [19-22].

\section{In vitro cell model}

RPE cells, originally obtained from ATCC, were exposed to USMB, with cells either in suspension or monolayer configurations. The cells were cultured in tissue culture flasks under $5 \% \mathrm{CO}_{2}$ at $37{ }^{\circ} \mathrm{C}$ in Ham's DMEM F12 medium, (Life technologies, Carlsbad, CA), supplemented with $10 \%(\mathrm{v} / \mathrm{v})$ fetal bovine serum and antibiotics $(10 \mathrm{mg} / \mathrm{mL}$ streptomycin and $66 \mu \mathrm{g} / \mathrm{mL}$ penicillin) and harvested by trypsinization. With the exception of microscopic studies of monolayers, the majority of the experiments described here were flow cytometric studies of cells treated while in suspension and fixed prior to flow cytometry. Cells were re-suspended at $10^{6}$ cells $/ \mathrm{mL}$ and treated with USMB in suspension. For cell monolayer studies, cells were seeded at $50 \%$ confluence on glass coverslips in 6-well plates for $24 \mathrm{~h}$ and subsequently treated with USMB.

Cells in suspension were treated with USMB and the release of molecules from cells was assessed using fluorescent markers and flow cytometry. The release of molecules was examined at $37{ }^{\circ} \mathrm{C}$ at $1.5,11.5$ or $21.5 \mathrm{~min}$ following the start of USMB. These time delays would be sufficient to observe the fast release from the lysosomes and the cytoplasm and the relatively slower release from early/recycling endosomes [13, 23]. Independent samples of cells were used to investigate the release of fluorescent markers from three cell compartments: (1) early/recycling endosomes, (2) cytoplasm, and (3) lysosomes for each time point. The earliest time point was $1.5 \mathrm{~min}$ which corresponds to $60 \mathrm{~s}$ of USMB treatment and $30 \mathrm{~s}$ of handling time until the samples are transferred on ice to stop the release of molecules. Subsequently, the cells were fixed with $4 \%$ PFA prior to flow cytometry analysis. The relative mean fluorescent intensity (MFI) of each fluorescent marker was measured using flow cytometry to indicate the amount of marker in cells treated or not with USMB. In addition, GFP-transfected RPE (RPE-GFP) cells (GFP fused to the cytosolic protein clathrin) were treated with USMB in a monolayer and the localization of bound LAMP-1 antibody at $1.5 \mathrm{~min}$ along with changes in GFP MFI were examined using confocal fluorescence microscopy.

\section{Release from early/recycling endosomes}

The release from early/recycling endosomes was investigated by loading RPE cells with $79 \mathrm{kDa}$ Alexa647Transferrin (Tfn) [24, 25]. Cells in suspension were loaded with Tfn by incubating with DMEM serum starvation media (D5796., Sigma-Aldrich Co., Oakville, $\mathrm{ON}, \mathrm{CA}$ ) for $60 \mathrm{~min}$, replacing the starvation media with PBS $^{3+}$ (Phosphate Buffered Saline containing $1 \mathrm{mM}$ $\mathrm{MgCl}_{2}, 1 \mathrm{mM} \mathrm{CaCl}$, and $5 \mathrm{mM}$ glucose) containing $20 \mu \mathrm{g} / \mathrm{mL}$ Tfn, and allowing the cells to internalize Tfn for $60 \mathrm{~min}$ at $37^{\circ} \mathrm{C}$. Excess Tfn was removed by centrifugation at $900 \mathrm{rpm}$ for $5 \mathrm{~min}$ at $4{ }^{\circ} \mathrm{C}$ and washing with $1 \mathrm{~mL}$ ice-cold PBS two times. Then, the cells were suspended in $37{ }^{\circ} \mathrm{CPBS}^{3+}$, exposed to USMB, and transferred to $4{ }^{\circ} \mathrm{C}$ to stop the release of the marker at each 
time point. Subsequently, the cells were washed with icecold PBS to remove the released marker, stained with a viability stain, and fixed to be analyzed. The MFI of Alexa647, which indicates the amount of Tfn inside the cell, was measured in untreated and USMB-treated samples.

\section{Release from the cytoplasm}

RPE-GFP cell samples were exposed to USMB, transferred to $4{ }^{\circ} \mathrm{C}$ at each time point, washed twice with ice-cold PBS to remove any released GFP, then stained with a viability stain, and fixed. After fixation, the MFI of GFP was measured to indicate the amount of GFP in the cytoplasm of the cell in untreated and USMB-treated samples.

\section{Release from lysosomes}

The release of lysosomal content was assessed using a Phycoerythrin (PE)-conjugated antibody against the lumemal/extracellular domain of LAMP-1 (A15798, Life technologies, Carlsbad, CA). Cells in suspension were treated with USMB then transferred to $4{ }^{\circ} \mathrm{C}$ to stop the release at each time point. The samples were washed with ice-cold PBS to remove any released material and transferred back on ice, stained with a viability stain, and fixed. The fixed cells were stained with LAMP-1 antibody $(400 \mu \mathrm{L} / \mathrm{mL})$ for $30 \mathrm{~min}$ at room temperature. Unbound antibody was removed by washing three times with PBS. The MFI of LAMP-1 antibody was measured using flow cytometry to indicate the amount LAMP-1 antibody binding. Increased LAMP-1 antibody binding indicates exposure of the lumen of lysosomes to the antibody which indicates either 1) lysosomal fusion with the cell membrane or 2) the permeabilization of both the plasma membrane and intracellular lysosomes.

\section{Cell viability}

Cell viability in the untreated control and in USMBtreated samples was measured using 7-Aminoactinomycin D (7-AAD), (559925, BD Biosciences, Mississauga, ON, $\mathrm{CA}$ ), in combination with all of the markers before fixation by incubating $5 \mu \mathrm{L}$ of $0.5 \mathrm{mg} / \mathrm{mL} 7$-AAD with the cell samples on ice for $30 \mathrm{~min}$. After incubating with 7 -AAD, the 7-AAD was removed from the medium by washing three times with ice-cold PBS. Then, the cells were fixed with $4 \%$ PFA in PBS for $15 \mathrm{~min}$ at room temperature. The incorporation of 7-AAD indicates that the membranes of these cells were not able to recover over the time period [26, 27]. Cells that do not incorporate 7 -AAD were considered viable cells, and 7-AAD positive cells were considered non-viable cells.

\section{The localization of bound LAMP-1 antibody in the cell} GFP-RPE cells in a monolayer were treated with USMB then placed on ice at $1.5 \mathrm{~min}$ from the start of the USMB treatment. The samples were washed twice with ice-cold PBS to remove any released cellular material, fixed with 4\% PFA in PBS for 15 min then stained with LAMP-1 antibody $(400 \mu \mathrm{L} / \mathrm{mL})$ for $30 \mathrm{~min}$ at room temperature. The coverslips were then mounted on slides and imaged with confocal fluorescence microscopy using a Leica TCS SL microscope (Leica TCS SL., Leica Microsystems Inc., Wetzlar, Germany) at 40× magnification.

\section{The subcellular localization of Tfn and LAMP-1}

RPE cells not expressing any fluorescent protein (parental $\mathrm{RPE}$ cells) in a monolayer were treated with $20 \mu \mathrm{g} / \mathrm{mL}$ Alexa555-conjugated transferrin (A555-Tfn) for $60 \mathrm{~min}$ at $37{ }^{\circ} \mathrm{C}$. The samples were washed twice with ice-cold PBS to remove any excess (unbound) A555-Tfn, fixed with 4\% PFA in PBS for $15 \mathrm{~min}$ and then permeabilized and stained with LAMP-1 antibody $(400 \mu \mathrm{L} / \mathrm{mL})$ for $60 \mathrm{~min}$ at room temperature. The coverslips were then mounted on slides and imaged using epifluorescence microscopy. The widefield epifluorescence microscopy images presented in Additional file 1: Figure S1 were obtained using a 63× (NA 1.49) oil objective on a Leica DM5000 B epifluorescence microscope using a DFC350FX camera (Leica DM5000 B., Leica Microsystems, Wetzlar, Germany). Indeed, Transferrin and LAMP-1 labeled two largely distinct membrane-bound intracellular compartments in the RPE cells used in this study (Additional file 1: Figure S1).

\section{USMB exposure}

Cells were exposed to $500 \mathrm{kHz}$ pulse centre frequency, $570 \mathrm{kPa}$ peak negative pressure (Pneg), $32 \mu$ s pulse duration (16 cycles tone burst) at $1 \mathrm{kHz}$ pulse repetition frequency (PRF) corresponding to $3.2 \%$ duty cycle, for $60 \mathrm{~s}$ in $37{ }^{\circ} \mathrm{C} \mathrm{PBS}^{3+}$. Immediately prior to ultrasound treatment of each sample, Definity mirobubbles (Lantheus Medical Imaging Inc., Saint-Laurent, QC, CA) were added. The Definity microbubbles were activated using a Vialmix for $45 \mathrm{~s}$. Using the cell suspension model, cells at $10^{6}$ cells $/ \mathrm{mL}$ were exposed to ultrasound using a single element focused transducer of $500 \mathrm{kHz}$ frequency, $9.2 \mathrm{~mm}-6 \mathrm{~dB}$ beam width, and $50 \mathrm{~mm}$ focal distance (IL0508HP, Valpey Fisher Inc, Hopkinton, MA, USA). A schematic diagram of the USMB exposure setup is shown in Fig. 1 (a). The setup consisted of an arbitrary waveform generator, connected to a power amplifier (AG series Amplifier, T\&C power conversion, Inc., NY, USA), which transmitted the electrical signal to the ultrasound transducer; the transducer was focused at the centre of the treatment chamber. A volume of $10 \mu \mathrm{L}$ of microbubbles was added to the $0.6 \mathrm{~mL}$ cell sample in the treatment chamber and a magnetic stirrer assured the mixing of cells and bubbles during the treatment. Using the monolayer model, cells were exposed to USMB using a $500 \mathrm{kHz}$ single element flat transducer with $32 \mathrm{~mm}$ element diameter focused at $85 \mathrm{~mm}$ and a $-6 \mathrm{~dB}$ beam width of 


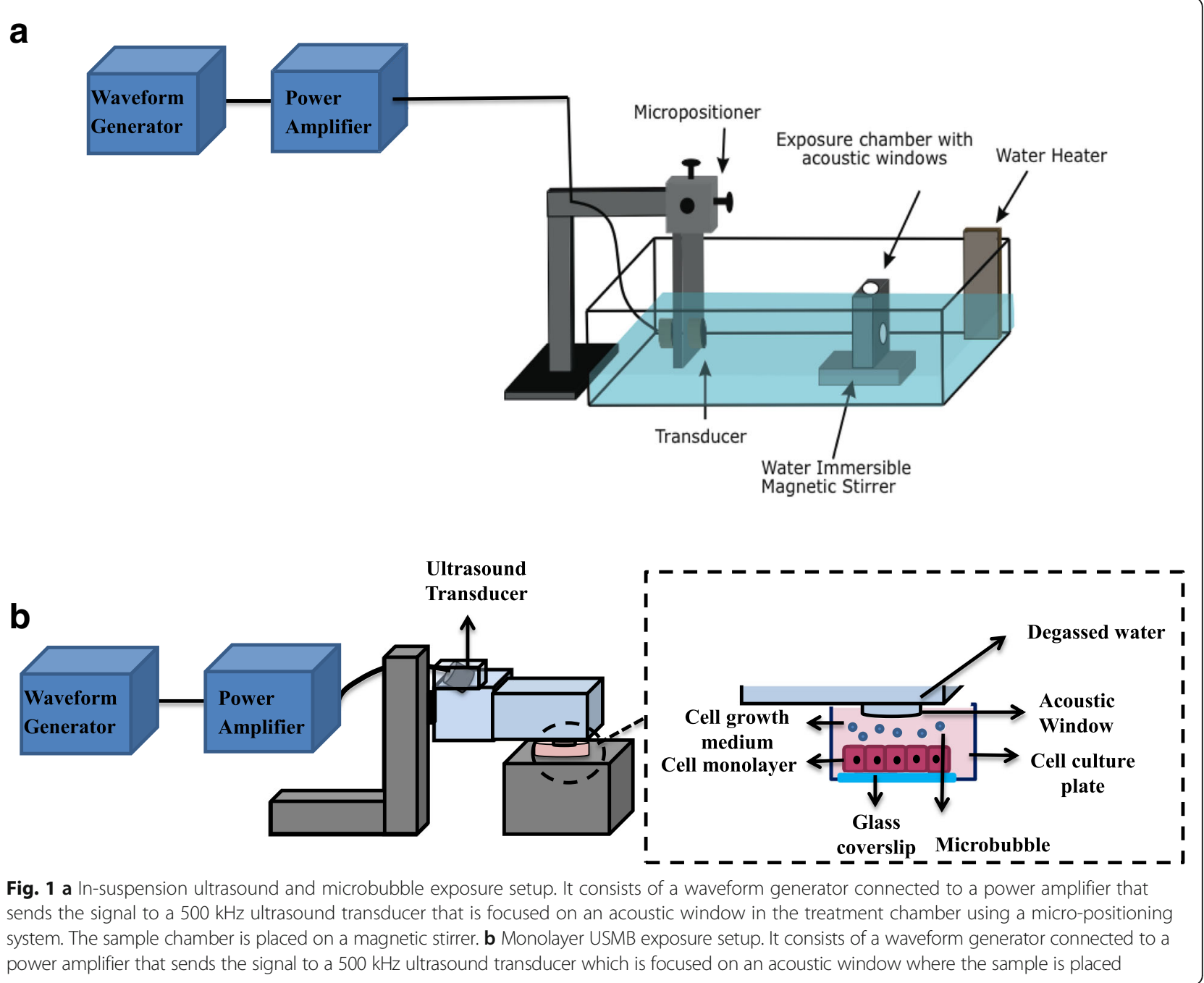

$31 \mathrm{~mm}$ at the focal point (IL0509GP., Valpey-Fisher Inc., Hopkinton, MA, USA) at a microbubble concentration of $10 \mu \mathrm{L} / \mathrm{mL}$. The wells of the six-well plates were filled with $13 \mathrm{~mL} \mathrm{PBS}^{3+}$. A schematic diagram of the monolayer USMB exposure setup is shown in Fig. 1 (b). The setup consisted of an arbitrary waveform generator, connected to a power amplifier (AG series Amplifier, T\&C power conversion, Inc., NY, USA), which transmitted the electrical signal to the ultrasound transducer. The transducer was submerged in partially degassed deionized water and focused obliquely at the centre of an acoustic window.

\section{Analysis}

A MACSQuant flow cytometer (MACSQuant ${ }^{\circ}$ Instrument., Miltenyi Biotec $\mathrm{GmbH}$, Bergisch Gladbach, Germany) was used in this study. A $488 \mathrm{~nm}$ laser was used to excite GFP, $\mathrm{PE}$ and 7-AAD and $638 \mathrm{~nm}$ laser was used to excite Alexa-647. The laser powers were $30 \mathrm{~mW}$ and $20 \mathrm{~mW}$ for the $488 \mathrm{~nm}$ and the $638 \mathrm{~nm}$ lasers, respectively.
Fluorescence emitted from GFP was collected using a $525 / 50 \mathrm{~nm}$ bandpass filter while a 585/40 $\mathrm{nm}$ bandpass filter was used for collecting PE fluorescence. A 750LP bandpass filter was used for 7-AAD and a $655-730 \mathrm{~nm}$ bandpass filter was used for Alexa-647. The instrument was configured for medium sample flow rate $(50 \mu \mathrm{L} / \mathrm{min})$. Data were acquired and analyzed using Macsquantify software. At the beginning of every acquisition session, a gate was set using a negative cell control to select single cell events. Of the single cell population, gates were set around the sub-populations of interest where the percentage of cells and the mean fluorescent intensity in each gate was recorded. In every experiment, a negative control of unstained RPE cells was used to identify the cell population in addition to a 7-AAD stained control of untreated cells. In addition, a positive control of 7-AAD stained cells was prepared by adding 7-AAD to cells permeabilized by fixation with PFA. In the analysis, single stained cell samples with Tfn-Alexa-647, GFP and LAMP-1 antibody were 
used. The MFI values of the markers in 10,000 cells from each sample were measured and were normalized to the MFI values for the untreated control at $t=1.5 \mathrm{~min}$. The number of samples was $n=4-6$ and Mann-Whitney $U$ non-parametric test was used to indicate statistically significant differences in MFI between groups. The MFI values of the USMB-treated groups were considered significantly higher than the untreated groups if the $p$-values associated with the one-tailed Mann-Whitney $U$ test were less than a significance level of $\alpha=0.05$. In addition, the MFI of GFP and LAMP-1 antibody from the fluorescent images acquired with the monolayer configuration were measured using ImageJ. Sixty cells from three independent trials per condition were examined from each group. The experimental conditions and the number of independent samples per condition are shown in Table 1.

\section{Results}

The biological effects of ultrasound and microbubble treatment, in particular with respect to the induction of release of cellular material by this treatment, remain poorly understood. To determine how USMB treatment impacts cellular release of material from the cytoplasm, endosomes and lysosomes, we have used specific markers of each compartment and assessed the release of each marker from cells upon exposure to USMB.

\section{Cell viability}

Figure 2 shows the percentage of viable cells in USMBtreated and untreated control samples at 1.5, 11.5 and $21.5 \mathrm{~min}$ after the start of USMB exposure normalized to the cell viability of the untreated control at $1.5 \mathrm{~min}$. As expected, USMB induced a decrease in cell viability. The percentage of viable cells decreased from $100 \pm 2 \%$ in the untreated control to $55 \pm 17 \%$ in the USMBtreated group at $1.5 \mathrm{~min}$. The percentages of viable cells in both the untreated controls and USMB-treated samples remained unchanged over the $\sim 20$ min duration; no statistically significant differences were observed.

\section{Release from early/recycling endosomes}

Figure 3 shows the MFI corresponding to intracellular (endosomal) Tfn in untreated control cells and USMBtreated cells at 1.5, 11.5 and $21.5 \mathrm{~min}$ with respect to the start of USMB exposure; the data were normalized to the MFI of the untreated control at $t=1.5 \mathrm{~min}$ of Tfn recycling. In the control cells, the reduction of MFI represents the basal rate of TfR recycling as it is undergoing constitutive endocytosis and recycling. Importantly, USMB significantly increased Tfn release from early/recycling endosomes immediately (at $1.5 \mathrm{~min}$ ) and the amount of intracellular Tfn remained lower than control up to $21.5 \mathrm{~min}$ in viable USMB-treated cells. The MFI of intracellular Tfn in USMB-treated viable cells decreased from $100 \%$ to $77 \%$, from $80 \%$ to $67 \%$, and from $50 \%$ to $46 \%$ at $1.5,11.5$ and $21.5 \mathrm{~min}$, respectively. In contrast, the MFI corresponding to A555-Tfn in non-viable USMB-treated cells was not statistically significant compared to the untreated control.

\section{Release from cytoplasm}

Figure 4 (a) shows the percentage of GFP positive cells $(\mathrm{GFP}+)$ in untreated control and USMB-treated samples at $1.5,11.5$ and $21.5 \mathrm{~min}$. USMB did not significantly affect the percentage of GFP+ cells after the treatment. All $(100 \%)$ of the cells were GFP+ before USMB and a total of $97 \%$ (including viable and non-viable cells) remained GFP+ after USMB. Upon USMB exposure, of the $97 \%$ that remained GFP+, $45 \%$ of the cells were GFP + and viable while $52 \%$ of the cells were GFP+ and nonviable. These percentages remained constant for $\sim 20$ min after USMB stimulation. Figure 4 (b) shows the MFI of GFP+ cells in untreated control and USMBtreated cells at 1.5, 11.5 and $21.5 \mathrm{~min}$; the data were normalized to the MFI of the untreated control at $1.5 \mathrm{~min}$. USMB caused an increase in the release of GFP from the cytoplasm, and the amount of GFP released was dependent on cell viability. The MFI decreased by $19 \%$ at $1.5 \mathrm{~min}$ in the viable USMB-treated group compared to the untreated control. In contrast to the small but statistically significant decrease in MFI of cytosolic GFP in viable cells, the MFI of cytosolic GFP in cells that became non-viable after USMB exposure decreased by robust $67 \%$ compared to the untreated control. The MFI decreased within $1.5 \mathrm{~min}$ from the start of USMB and did not significantly change with time. This indicates that upon USMB treatment, the majority of the loss of cytosolic material occurs in cells that are non-viable, while a smaller but significant release of cytosolic material occurs in cells that remain viable after USMB treatment. The effect of USMB on the release of cytosolic material from cells appears to be immediate (within $1.5 \mathrm{~min}$ ) and does not significantly change with the time delay after USMB.

Table 1 Experimental conditions and number of independent samples per condition

\begin{tabular}{lllllll}
\hline & $1.5 \mathrm{~min} / \mathrm{no}$ USMB & $11.5 \mathrm{~min} / \mathrm{no}$ USMB & $21.5 \mathrm{~min} / \mathrm{no}$ USMB & $1.5 \mathrm{~min} /$ USMB & $11.5 \mathrm{~min} /$ USMB & $21.5 \mathrm{~min} /$ USMB \\
\hline Tfn +7-AAD & $n=4$ & $n=4$ & $n=4$ & $n=4$ & $n=4$ & $n=4$ \\
GFP + 7-AAD & $n=4$ & $n=4$ & $n=4$ & $n=6$ & $n=6$ & $n=6$ \\
LAMP-1 + 7-AAD & $n=4$ & $n=4$ & $n=4$ & $n=4$ & $n=4$ & $n=4$ \\
\hline
\end{tabular}




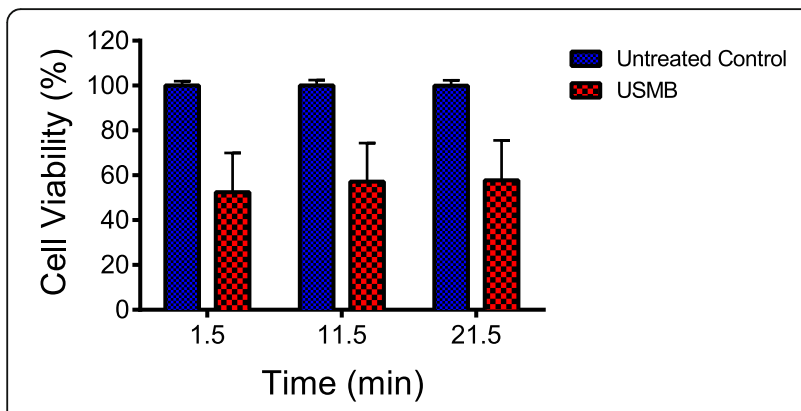

Fig. 2 The percentage of viable cells in ultrasound and microbubble (USMB) treated and untreated control at 1.5, 11.5, and $21.5 \mathrm{~min}$ assessed using 7-Aminoactinomycin D (7-AAD). The number of samples is $n=12$ for the untreated group and $n=14$ for the USMB treated group. The error bars represent the standard deviation

\section{Release from lysosomes}

Figure 5 shows the MFI of LAMP-1 antibody staining (without permeabilization by detergents) in untreated control and USMB-treated cells at 1.5, 11.5 and $21.5 \mathrm{~min}$. All groups were normalized to the MFI of LAMP-1 antibody staining of the untreated control cells. USMB increased the binding of LAMP-1 antibody in cells as indicated by the increase in the MFI of the LAMP-1 antibody label in the USMB-treated cells at 1.5 min compared to the untreated control. However, this increase is highly dependent on cell viability. When compared to the untreated control, USMB induced a $50 \%$ increase in LAMP-1 antibody MFI in viable cells immediately after USMB. In stark contrast, USMB induced a dramatic increase in MFI in non-viable cells; the MFI increased by 15 fold compared to the untreated control. The USMB induced lysosome exocytosis (in viable cells) and the large increase in LAMP-1 antibody labeling (in non-viable cells) appeared to be immediate and remained constant over $21.5 \mathrm{~min}$.

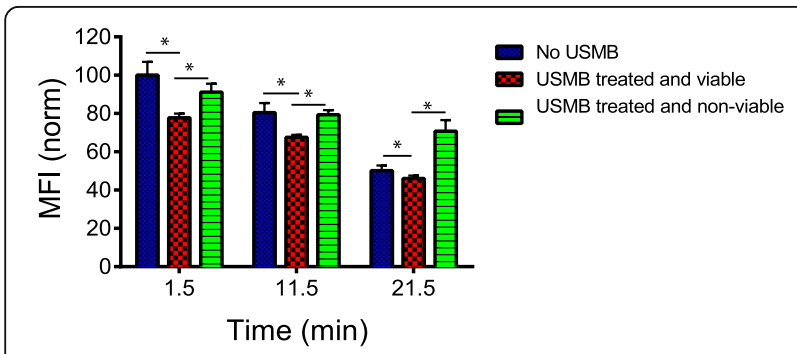

Fig. 3 The mean fluorescent intensity (MFI) in untreated controls and ultrasound and microbubble (USMB) treated Alexa647-Transferrin (Tfn) loaded cells at 1.5, 11.5, and $21.5 \mathrm{~min}$. The number of samples is $n=4$ for all groups and the $\left(^{*}\right)$ indicates statistical significance ( $p<0.05)$ using a Mann-Whitney $U$ test. The error bars represent the standard deviation

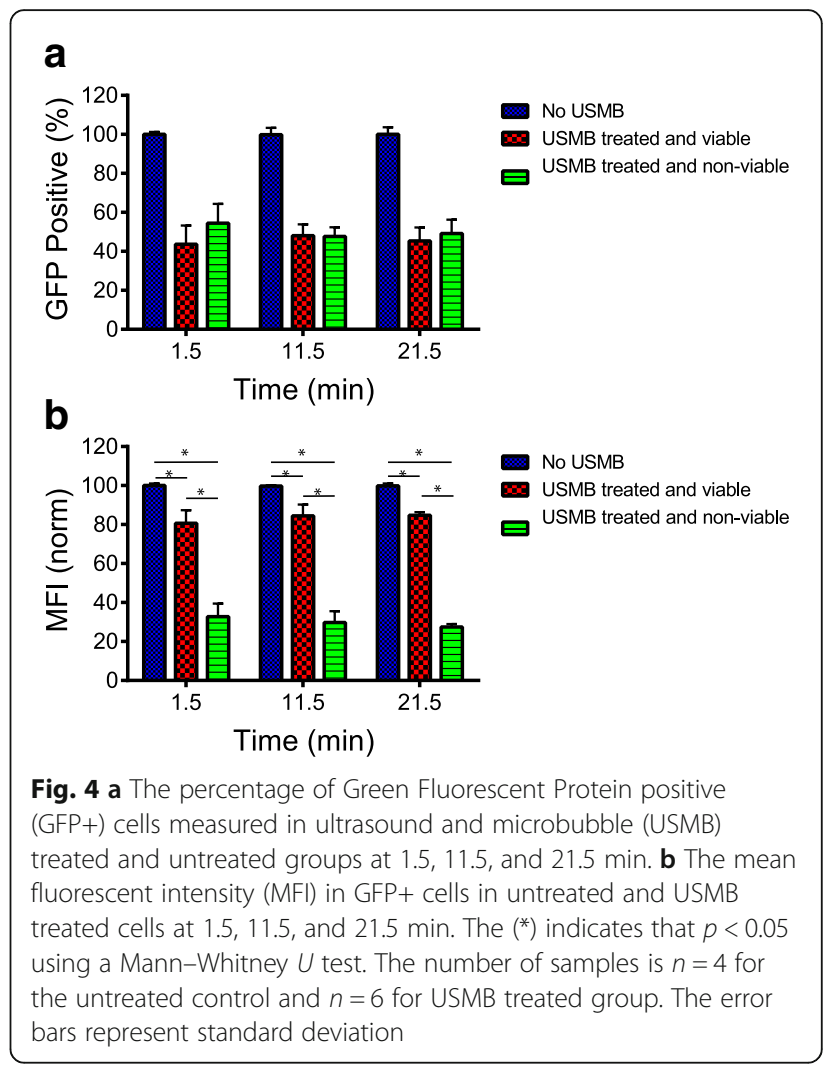

Relationship between the release from cytoplasm and lysosomes

Figure 6 shows the relationship between the MFI of GFP and the MFI of LAMP-1 antibody in untreated and USMB-treated cells. The MFI values for each marker were obtained from independent experiments and normalized to the $1.5 \mathrm{~min}$ MFI values of the untreated control. There appears to be a correlation between the MFI of GFP (cytosolic marker) and the MFI of LAMP-1 antibody labeling in cells and this correlation appears to be dependent on cell viability. As the cell's GFP MFI

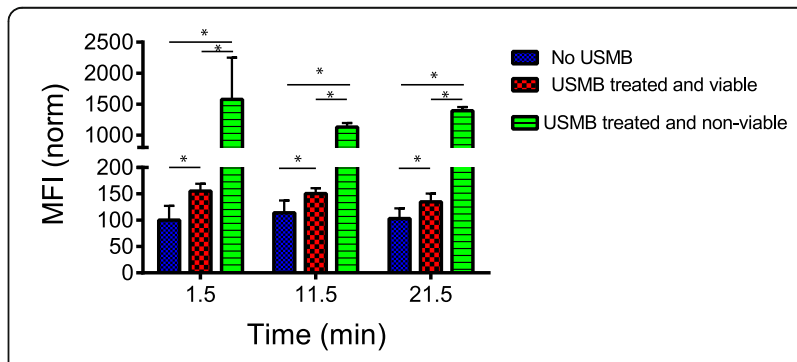

Fig. 5 The mean fluorescent intensity (MFI) from Lysosomal Associated Membrane Protein-1. (LAMP-1) antibody binding measured in untreated control and ultrasound and microbubble (USMB) treated samples at 1.5, 11.5 , and $21.5 \mathrm{~min}$ from the start of USMB. The $\left(^{*}\right)$ indicates a statistically significant difference $(p<0.05)$ using a Mann-Whitney $U$ test. The number of samples $n=4$ for both USMB treated and untreated groups. The error bars represent the standard deviation 


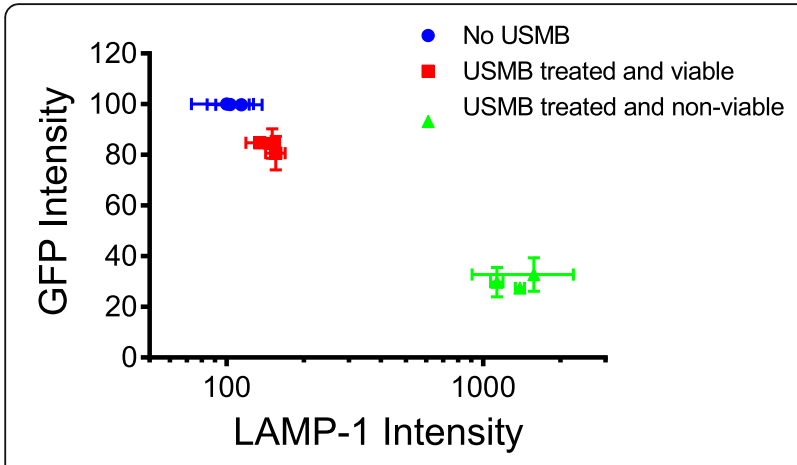

Fig. 6 The relationship between the release from cytoplasm and lysosomes obtained by plotting the mean fluorescent intensities (MFI) of Green Fluorescent Protein (GFP) and Lysosomal Associated Membrane Protein-1 (LAMP-1) antibody. The MFI values for each marker were obtained from independent experiments and were normalized to the MFI values of the untreated control at $t=1.5 \mathrm{~min}$ for each marker. The error bars represent standard deviation

decreases, there is an increase in LAMP-1 antibody binding. A $19 \%$ decrease in GFP MFI in cells that remain viable after USMB is associated with a $50 \%$ increase in LAMP-1 antibody labeling. However, in non-viable cells, a $67 \%$ decrease in GFP MFI is associated with a 15 -fold increase in LAMP-1 antibody labeling.

\section{The localization of binding LAMP-1 antibody in the cell}

The robust increase in LAMP-1 antibody labeling in cells that became non-viable upon USMB compared to that seen in cells that remained viable could be due to a much higher amount of lysosomal exocytosis in nonviable cells as these cells are attempting to repair their damaged membranes (within $1.5 \mathrm{~min}$ from the start of USMB), or could reflect partial permeabilization of lysosomal membranes (along with permeabilization of the plasma membrane) that correlates with loss of cell viability. To distinguish between these possibilities, the subcellular localization of LAMP-1 antibody staining was examined using immunofluorescence microscopy. Figure 7 shows the fluorescent images of cells expressing the cytosolic GFP marker and stained with LAMP-1 antibodies, as individual channels as well as their merged images, for untreated-control and USMB-treated cells. USMB-treated cells have significantly more LAMP-1 antibody labeling compared to the untreated control and this antibody labeling appears to be in well-defined punctate structures, consistent with LAMP-1 antibody labeling of intracellular structures, such as lysosomes, and not primarily on the cell surface (Fig. 7 (a) and (b)). Additionally, in the USMB treated cells, the labeling of cells with LAMP-1 antibody is highly heterogeneous, with some cells having more LAMP-1 antibody binding than others. Furthermore, cells that exhibit substantial
LAMP-1 antibody staining appear to have lower GFP levels (cytosolic marker) compared to cells that exhibit lower levels of LAMP1 antibody staining, as shown in Fig. 7 (c) and (d). The merged images of LAMP-1 and GFP for untreated and USMB-treated cells are shown in Fig. 7 (e) and (f), respectively. The merged images show the dispersed distribution of GFP throughout the cytoplasm of the cell and the localization of LAMP-1 antibody in punctuate intracellular structures suggesting the permeabilization of intracellular lysosomes upon USMB exposure.

\section{Discussion}

The efficacy by which USMB potentiates therapies involving the cellular uptake of biologically active molecules depends on the induction of enhanced uptake and minimal release from cells. In this study, it was demonstrated that USMB increased the release of fluorescent markers from specific cell compartments including the cytoplasm, lysosomes and early/recycling endosomes, and that this effect was highly dependent on cell viability. This could be an indication that the USMBenhanced release of molecules is directly related to the membrane damage induced by USMB $[11,13]$. USMB induced a decrease in cell viability and this is consistent with previous studies that show the effects of USMB on cell viability $[6,28]$. This decrease in cell viability after USMB is the result of irreversible membrane disruption induced by USMB that allows 7-AAD to enter the cell and bind to the DNA [7].

Tfn release from early/recycling endosomes was significantly increased by USMB at $1.5 \mathrm{~min}$ in USMBtreated viable cells compared to the untreated control. However, this increase in recycling was not statistically significant in cells that were non-viable after USMB. Studies have suggested that the release of Tfn from endosomes is not driven by the increased calcium influx into the cell after membrane disruption [29]. Therefore, it can be suggested that the underlying mechanism of the increased release from early/recycling endosomes could be associated with an active, regulated cellular mechanism as a response to the biomechanical stress induced by USMB. This active cellular mechanism may be the result of enhanced endomembrane traffic flux, as USMB also enhances endocytosis, specifically clathrin mediated endocytosis which is the pathway of Tfn uptake or it may be in response to promoting plasma membrane repair [8-10]. Following internalization, Tfn is well known to populate several endosomal membrane compartments which have distinct recycling kinetics. For instance, recycling from an early endosomal subpopulation has $t_{1 / 2}$ for recycling of $\sim 2 \mathrm{~min}$, while recycling from the perinuclear recycling endosomes has a $t_{1 / 2}$ for recycling on the order of $10-20 \mathrm{~min}([30])$. The results 


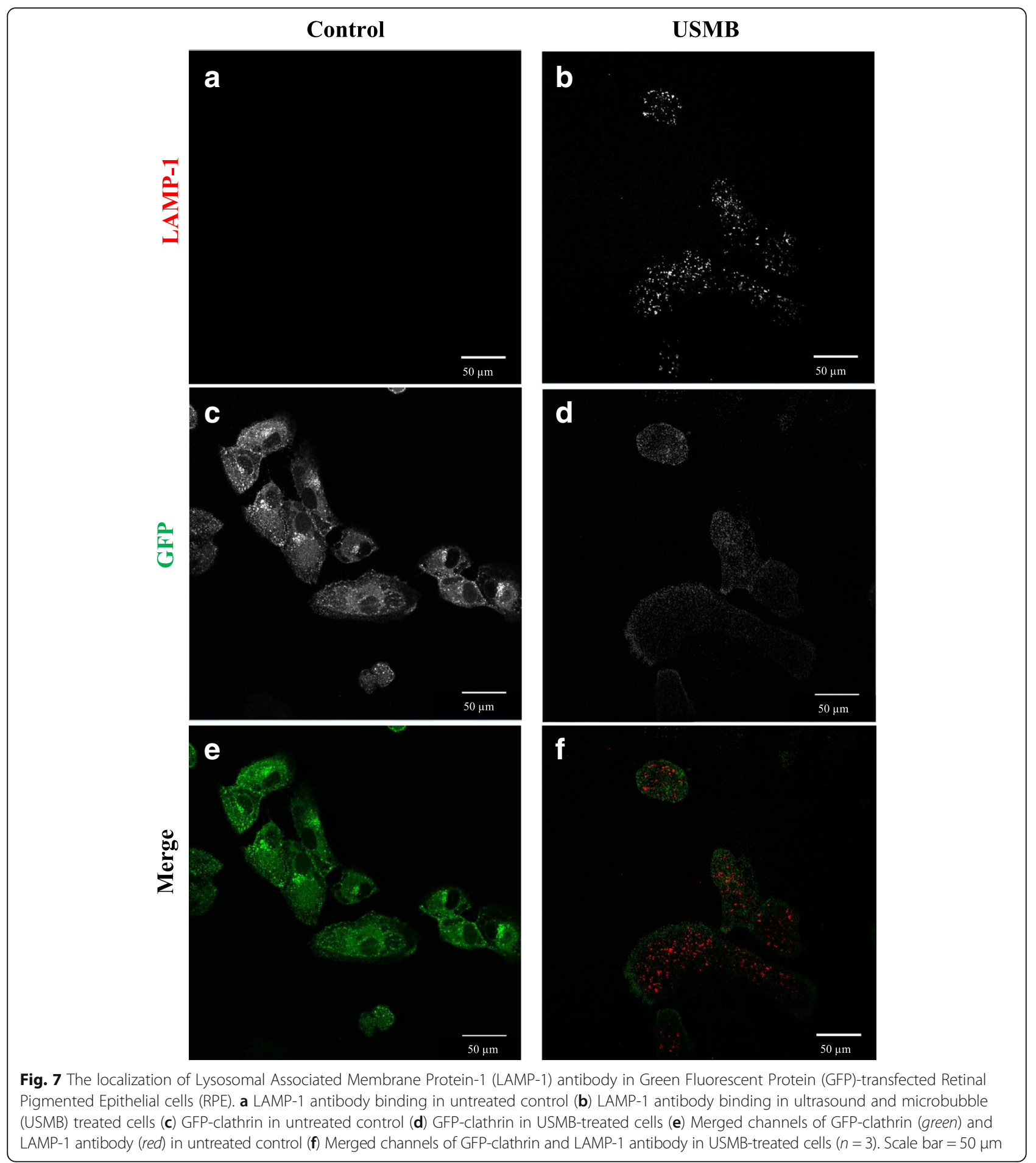

of this study indicate that USMB may selectively trigger enhanced exocytosis from early, fast recycling endosomes. As such, after the initial burst of Tfn recycling in the $1.5 \mathrm{~min}$ following the start of USMB, Tfn recycling from these cells may be largely limited to that from perinuclear, slow recycling endosomal compartments. In contrast, cells not stimulated with USMB exhibit a constant rate of recycling from both fast and slow recycling endosomal compartments. This model readily explains why the recycling that occurs after the initial burst 
induced by USMB treatment in viable USMB-treated cells (in the period from 1.5 to $21.5 \mathrm{~min}$ ) appears slower than in control cells, such that the difference in total cellular fluorescent transferrin decreases between control and USMB-treated cells over time. Additionally, the slower overall observed rate of Tfn recycling in cells which remained viable after USMB could be related to the oxidative stress induced by USMB through the production of reactive oxygen species (ROS) [31, 32] or due to depolarization and local hyperpolarization of the plasma membrane [33]. Furthermore, there was minimal release of Tfn in non-viable cells upon USMB treatment, in particular at 1.5 and 11.5 min following USMB treatment, indicating that USMB does not cause a nonspecific permeabilization of intracellular membranes. Instead, non-viable cells largely retain Tfn intracellularly within endomembranes that fail to undergo exocytosis.

USMB induced the release from the cytoplasm of cells and this release was also found to be dependent on cell viability. Although USMB induced the release from the cytoplasm in both viable and non-viable cells, a much higher amount of cytoplasmic content was released from cells that became non-viable compared to cells that remained viable following USMB. Importantly, by comparing the release of cytoplasmic content in viable versus non-viable cells, this study identified that when measuring cytoplasmic release from cells upon USMB treatment, non-viable cells contribute much more to the measurement of cytoplasmic release than do viable cells. Hence, when studying the effects of USMB treatment on release of materials from the cytoplasm, it is important to distinguish between viable cells (that presumably rapidly close pores formed during sonoporation) and those that are non-viable (that fail to repair membrane damage caused by USMB treatment). This USMB-induced GFP release from the cytoplasm is consistent with the previously reported effect of USMB-induced release of GFP from the cytoplasm of the cell immediately following USMB exposure [11]. In addition, under our exposure conditions, the percentage of GFP+ cells remained consistent following USMB in contrast to a previous study where a decrease in GFP+ cells observed following USBM was concurrent with an increase in PI+ cells [11].

An increase in the detection of lysosomal content from the outside of the cell was observed after USMB and this increase was higher in non-viable cells compared to viable cells. In viable cells, this increase in detection of LAMP-1 likely reflects an increase in lysosome exocytosis upon USMB treatment. Cytosolic calcium levels were found to increase after sonoporation by the leaking of calcium into the cell with its concentration gradient through USMB-induced membrane disruptions [34]. The increased lysosomal exocytosis induced by calcium influx is due to the calcium-sensitive synaptotagmin VII membrane tethering protein on lysosomal membranes $[13,16]$. Lysosomal exocytosis by complete fusion with the plasma membrane is thought to be the main mechanism of membrane repair after sonoporation [13]. There are two hypothesized mechanisms of membrane repair by lysosomal exocytosis: (1) lysosomes form a patch to repair the damaged site or (2) lysosomes deliver extra membrane to the cell surface to reduce membrane tension and help close the pores [13].

In non-viable cells, the increased detection of lysosomal content from the cell exterior could be a result of exceptionally high levels of lysosomal fusion with the cell membrane as a mechanism for membrane repair $[13,35]$ or due to permeabilization of lysosomal membranes inside the cell [36]. That non-viable cells detected after USMB exhibit a large increase of punctate lysosomal signal and not a uniform signal provides evidence for the latter. Since the plasma membrane permeabilization induced by USMB is sufficient to allow efflux of small proteins, such as GFP, but not of organelles like lysosomes, permeabilization of both the plasma membrane and the lysosomes are needed in order for LAMP-1 antibody to access the lumen of lysosomes. This permeabilization of lysosomes in non-viable cells appears to be selective to this organelle, since a similar permeabilization of early/recycling endosomes was not observed in this cell population (as seen by the intracellular retention of transferrin in non-viable cells upon USMB treatment). Hence, it is unlikely that the increased permeabilization of lysosomes following USMB treatment merely reflects loss of plasma membrane integrity upon loss of cell viability, but instead may indicate that the lysosomal membranes may also be subject to permeabilization upon USMB. It is possible that excessive pore formation in lysosomes upon USMB treatment may contribute to loss of cell viability in some cells. The permeabilization of lysosomal membranes could be a result of oxidative stress generated by cellular treatment with USMB. Under the exposure conditions of this study which corresponds to a mechanical index (MI) of 0.8 , the acoustic mechanism associated with enhanced leakage is inertial cavitation. Microbubbles exposed to high acoustic pressures and low pulse centre frequencies undergo inertial cavitation [37]. In addition, inertial cavitation has been shown to increase the temperature locally resulting in breaking of chemical bonds and the production of ROS including hydrogen peroxide $\left(\mathrm{H}_{2} \mathrm{O}_{2}\right)$ [34]. Oxidative stress as a result of increased intracellular levels of $\mathrm{H}_{2} \mathrm{O}_{2}$ was found to induce permeabilization of lysosomal membranes inside the cell and this disruption of lysosomes is thought to be a signal for cell death [36]. Figure 8 summarizes the hypothesized mechanisms of USMB induced or enhanced release from cytoplasm, lysosomes, and early/recycling 


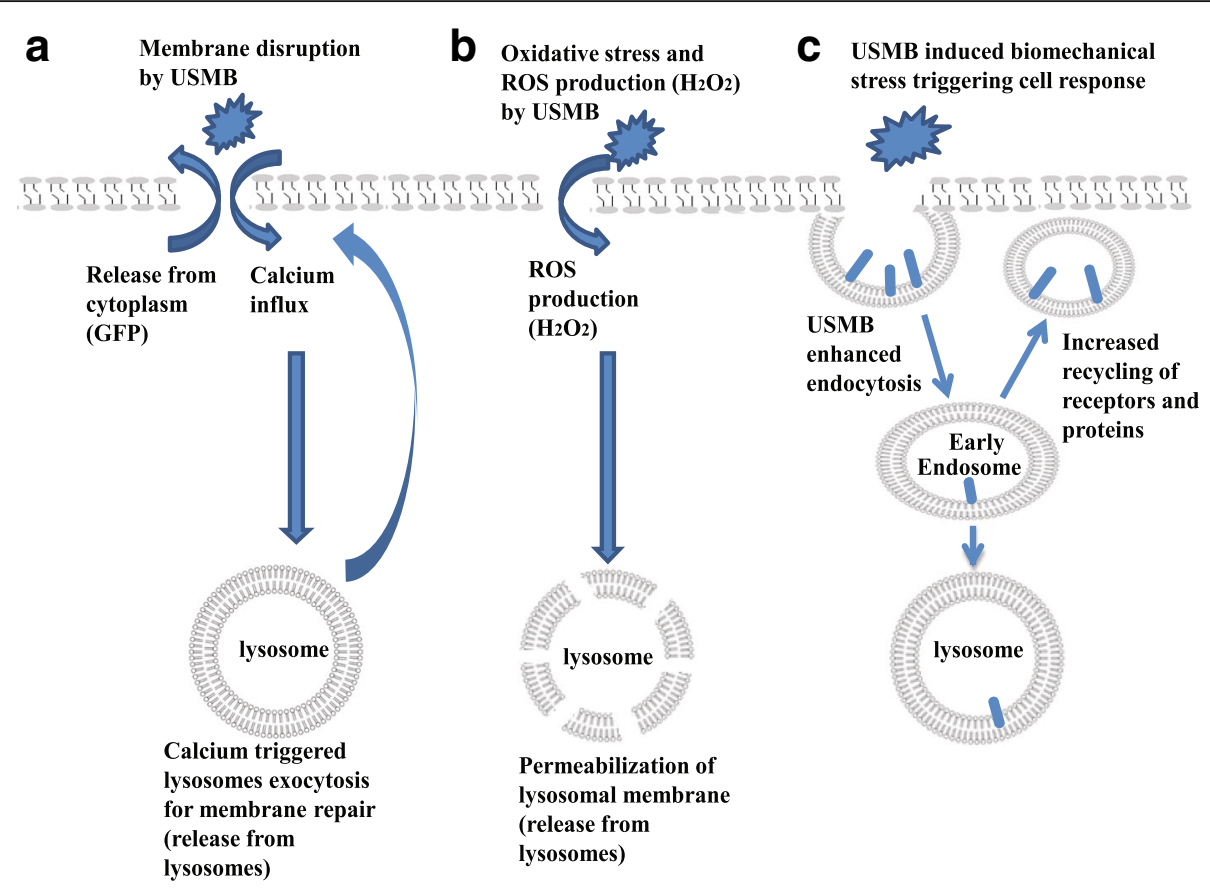

Fig. 8 A schematic diagram summarizing the hypothesized mechanisms of USMB induced/enhanced release from cytoplasm, lysosomes, and early/recycling endosomes through both membrane disruption and exocytosis. These mechanisms can occur simultaneously and can affect the release from more than one compartment at the same time (a) Membrane disruption by USMB induces diffusion of molecules from the cytoplasm and causes an increase in $\mathrm{Ca}^{2+}$ influx which triggers lysosomes fusion for membrane repair and can also be involved in USMB enhanced endocytosis. b USMB induced production of $\mathrm{H}_{2} \mathrm{O}_{2}$ can induce the permeabilization of lysosomal membranes and the release of lysosomal content and this oxidative stress can also be involved in the slower rate of recycling from early/recycling endosomes (c) USMB induced biomechanical stress can cause enhancing endocytosis and triggering a cellular response driving release from early/recycling endosomes

endosomes through both membrane disruption and exocytosis.

There are several limitations associated with the present study. The study did not examine the effects of varying treatment conditions such as microbubble concentrations and ultrasound pressure on the USMB enhanced release. Furthermore, it was assumed that ultrasound exposure without microbubbles has a minimal effect on the release of molecules from cells [11]. In addition, the 7-AAD viability marker stains cells whose membrane integrity has been compromised and does not assess the cell's ability to proliferate and form a colony. However, this study improved our understanding of the limitations of USMB drug therapy, as it showed the effect of USMB on drug retention in cells. Additionally, the effect of USMB on the release of cellular content may shed the light on new applications of USMB as a possible way of extracting biologically active molecules from cells to further study them [11] or as a tool to increase the release of biomarkers from cancer cells to the blood stream for better cancer detection and localization $[37,38]$.

\section{Conclusions}

USMB enhanced the release of molecules from early/recycling endosomes, cytoplasm and increased access to lysosomal content from the cell exterior through both membrane disruption and enhanced exocytosis. USMB enhanced the release of molecules in viable and non-viable cells to differing extents. A higher release of molecules from the cytoplasm and access to lysosomal content was observed in cells that were non-viable after USMB compared to cells that remained viable after the treatment and to the untreated control, suggesting that excessive release of cytoplasm or access to lysosomal content may be related to cell death upon USMB treatment. In contrast, a higher release of the contents from early/recycling endosomes was observed in viable cells compared to USMB-treated non-viable cells and to the untreated control, indicating that increased endomembrane recycling upon USMB was due to regulated, active processes stimulated by this treatment. In addition, the USMB induced release from the cytoplasm and extracellular access to lysosomal content appears to be immediate and independent of the time delay following USMB. Furthermore, LAMP-1 antibody labeling suggested the selective disruption of lysosomal membranes inside the cell after USMB treatment. 


\section{Additional file}

Additional file 1: Figure S1. Transferrin-loaded endosomes and LAMP-1 positive lysosomes are largely distinct compartments. RPE cells (not expressing any endogenous fluorescent proteins) were incubated with $10 \mu \mathrm{g} / \mathrm{mL}$ Alexa-555 conjugated transferrin (A555-Tfn) for $1 \mathrm{~h}$ at 37C. Subsequently, cells were rapidly washed, fixed, permeabilized, and subjected to immunofluorescence staining to detect endogenous LAMP-1. Cells were then further processed and subjected to widefield epifluorescence microscopy. Shown are two sets of representative micrographs of cells (under identical conditions) showing A555-Tfn (red), LAMP-1 (green), DAPI (blue) and merged images. These images showing little appreciable overlap of LAMP-1 and A555-Tfn signals. Scale $=10 \mu \mathrm{m}$. (PDF $4254 \mathrm{~kb}$ )

\section{Abbreviations}

7-AAD: 7-Aminoactinomycin D; A555-Tfn: Alexa555-conjugated transferrin GFP: Green fluorescent protein; GFP+: Green fluorescent protein positive cells; LAMP-1: Lysosomal associated membrane protein-1; MFI: Mean fluorescent intensity; MI: Mechanical index; PE: Phycoerythrin; $P_{\text {neg }}$ : Peak negative pressure; PRF: Pulse repetition frequency; ROS: Reactive oxygen species; RPE: Retinal pigmented epithelial cells; Tfn: Alexa646-Transferrin; USMB: Ultrasound and microbubbles

\section{Acknowledgement}

This work was funded by RK's NSERC Discovery grant.

\section{Availability of data and materials}

The data is not shared in this study.

\section{Authors' contributions}

FH carried out the experiments, analyzed the flow cytometry and fluorescent microscopy data, statistical analysis, and participated in the drafting and revising of the manuscript. CA participated in the design of the study, analysis of the data, and revising the manuscript critically. RK conceived the study, participated in the design and coordination of the study, the statistical analysis and final completion of the manuscript. All the authors read and approved the final manuscript.

\section{Competing interests}

The authors declare that they have no competing interests.

\section{Consent for publication}

Not applicable.

\section{Ethics approval and consent to participate}

Not applicable.

\section{Publisher's Note}

Springer Nature remains neutral with regard to jurisdictional claims in published maps and institutional affiliations.

\section{Author details}

'Department of Physics, Ryerson University, 350 Victoria Street Toronto, Ontario M5B 2K3, Canada. ${ }^{2}$ Department of Chemistry and Biology, Ryerson University, Toronto, Ontario, Canada. ${ }^{3}$ Institute for Biomedical Engineering, Science and Technology (iBEST), Toronto, Canada. ${ }^{4}$ Keenan Research Centre, St. Michael's Hospital, Toronto, Canada.

\section{Received: 1 April 2016 Accepted: 9 May 2017}

\section{Published online: 18 May 2017}

\section{References}

1. Krishan A, Fitz CM, Andritsch I. Drug retention, efflux, and resistance in tumor cells. Cytometry. 1997;29(4):279-85.

2. Antonescu CN, McGraw TE, Klip A: Reciprocal Regulation of Endocytosis and Metabolism. Cold Spring Harbor Perspectives in Biology. 2014;6(7):a016964.

3. Gundelfinger ED, Kessels MM, Qualmann B. Temporal and spatial coordination of exocytosis and endocytosis. Nat Rev Mol Cell Biol. $2003 ; 4(2): 127-39$
4. Qin $P, X u L, H u$ YX, Zhong WJ, Cai P, Du LF, Jin LF, Yu ACH. SonoporationInduced Depolarization of Plasma Membrane Potential: Analysis of Heterogeneous Impact. Ultrasound Med Biol. 2014;40(5):979-89.

5. Hu YX, Wan JMF, Yu ACH. Membrane Perforation and Recovery Dynamics in Microbubble-Mediated Sonoporation. Ultrasound Med Biol. 2013;39(12):2393-405.

6. Karshafian R, Bevan P, Williams R, Samac S, Burns PN. Sonoporation by Ultrasound-Activated Microbubble Contrast Agents: Effect of Acoustic Exposure Parameters on Cell Membrane Permeability and Cell Viability. Ultrasound Med Biol. 2009:35(5):847-60.

7. Karshafian R, Samac S, Bevan P, Burns P. Microbubble mediated sonoporation of cells in suspension: Clonogenic viability and influence of molecular size on uptake. Ultrasonics. 2010. doi:10.1016/j.ultras.2010.01.009.

8. Meijering BDM, Juffermans LJM, van Wamel A, Henning RH, Zuhorn IS, Emmer M, Versteilen AMG, Paulus WJ, van Gilst WH, Kooiman K, et al. Ultrasound and Microbubble-Targeted Delivery of Macromolecules Is Regulated by Induction of Endocytosis and Pore Formation. Circ Res. 2009;104(5):679-87.

9. Afadzi M, Strand SP, Nilssen EA, Masoy SE, Johansen TF, Hansen R, Angelsen BA, Davies CD. Mechanisms of the Ultrasound-Mediated Intracellular Delivery of Liposomes and Dextrans. leee Transactions on Ultrasonics Ferroelectrics and Frequency Control. 2013;60(1):21-33.

10. Fekri F, Delos Santos RC, Karshafian R, Antonescu CN. Ultrasound Microbubble Treatment Enhances Clathrin-Mediated Endocytosis and FluidPhase Uptake through Distinct Mechanisms. Plos One. 2016;11(6):e0156754.

11. Kaddur K, Lebegue L, Tranquart F, Midoux P, Pichon C, Bouakaz A. Transient transmembrane release of green fluorescent proteins with sonoporation. IEEE Trans Ultrason Ferroelectr Freq Control. 2010;57(7):1558-67.

12. Khanna S, Amso NN, Paynter SJ, Coakley WT. Contrast agent bubble and erythrocyte behavior in a 1.5-MHz standing ultrasound wave. Ultrasound Med Biol. 2003:29(10):1463-70.

13. Yang F, Gu N, Chen D, Xi XY, Zhang D, Li YX, Wu JR. Experimental study on cell self-sealing during sonoporation. J Control Release. 2008;131(3):205-10.

14. Zhou Y, Kumon RE, Cui J, Deng CX. The Size of Sonoporation Pores on the Cell Membrane. Ultrasound Med Biol. 2009;35(10):1756-60.

15. Baravalle G, Schober D, Huber M, Bayer N, Murphy RF, Fuchs R. Transferrin recycling and dextran transport to lysosomes is differentially affected by bafilomycin, nocodazole, and low temperature. Cell Tissue Res. 2005:320(1):99-113.

16. Idone $\mathrm{V}$, Tam C, Andrews NW. Two-way traffic on the road to plasma membrane repair. Trends Cell Biol. 2008;18(11):552-9.

17. Reddy A, Caler EV, Andrews NW. Plasma membrane repair is mediated by Ca(2+)-regulated exocytosis of lysosomes. Cell. 2001;106(2):157-69.

18. Lamb JE, Ray F, Ward JH, Kushner JP, Kaplan J. Internalization and subcellular localization of transferrin and transferrin receptors in HeLa cells. J Biol Chem. 1983;258(14):8751-8.

19. Winterstein C, Trotter J, Kramer-Albers EM. Distinct endocytic recycling of myelin proteins promotes oligodendroglial membrane remodeling. J Cell Sci. 2008;121(Pt 6):834-42.

20. Wilson R, Bate C, Boshuizen R, Williams A, Brewer J. Squalestatin alters the intracellular trafficking of a neurotoxic prion peptide. BMC Neurosci. 2007:8:99.

21. Chibalina MV, Seaman MN, Miller CC, Kendrick-Jones J, Buss F. Myosin VI and its interacting protein LMTK2 regulate tubule formation and transport to the endocytic recycling compartment. J Cell Sci. 2007:120(Pt 24):4278-88.

22. Burgdorf S, Scholz C, Kautz A, Tampe R, Kurts C. Spatial and mechanistic separation of cross-presentation and endogenous antigen presentation. Nat Immunol. 2008;9(5):558-66.

23. Hao MM, Maxfield FR. Characterization of rapid membrane internalization and recycling. J Biol Chem. 2000;275(20):15279-86.

24. Miller SE, Mathiasen S, Bright NA, Pierre F, Kelly BT, Kladt N, Schauss A, Merrifield CJ, Stamou D, Höning S, et al. CALM regulates clathrin-coated vesicle size and maturation by directly sensing and driving membrane curvature. Dev Cell. 2015;33(2):163-75.

25. Mayle KM, Le AM, Kamei DT. The intracellular trafficking pathway of transferrin. BBA-Gen Subjects. 2012;1820(3):264-81.

26. Schmid I, Krall WJ, Uittenbogaart CH, Braun J, Giorgi JV. Dead cell discrimination with 7-amino-actinomycin $\mathrm{D}$ in combination with dual color immunofluorescence in single laser flow cytometry. Cytometry. 1992:13(2):204-8.

27. Falzone N, Huyser C, Franken DR. Comparison between propidium iodide and 7-amino-actinomycin-D for viability assessment during flow cytometric analyses of the human sperm acrosome. Andrologia. 2010;42(1):20-6. 
28. Lai C-Y, Wu C-H, Chen C-C, Li P-C. Quantitative relations of acoustic inertial cavitation with sonoporation and cell viability. Ultrasound Med Biol. 2006:32(12):1931-41.

29. McNeil PL, Steinhardt RA. Plasma membrane disruption: repair, prevention, adaptation. Annu Rev Cell Dev Biol. 2003;19:697-731.

30. Mukherjee S, Maxfield FR. Membrane domains. Annu Rev Cell Dev Biol. 2004;20:839-66

31. Kooiman K, van der Steen AFW, de Jong N. Role of Intracellular Calcium and Reactive Oxygen Species in Microbubble-Mediated Alterations of Endothelial Layer Permeability. leee Transactions on Ultrasonics Ferroelectrics and Frequency Control. 2013;60(9):1811-5.

32. Malorni W, losi F, Santini MT, Rivabene R, Testa U. Oxidative Stress and Transferrin Receptor Recycling. Cytotechnology. 1993;11:53-5.

33. Juffermans LJ, Kamp O, Dijkmans PA, Visser CA, Musters RJ. Low-Intensity Ultrasound-Exposed Microbubbles Provoke Local Hyperpolarization of the Cell Membrane Via Activation of BKCa Channels. Ultrasound Med Biol. 2007;34(3):502-8.

34. Juffermans LJ, Dijkmans PA, Musters RJ, Visser CA, Kamp O. Transient permeabilization of cell membranes by ultrasound-exposed microbubbles is related to formation of hydrogen peroxide. Am J Physiol Heart Circ Physiol. 2006;291(4):H1595-1601.

35. Idone V, Tam C, Goss J, Toomre D, Pypaert M, Andrews N. Repair of injured plasma membrane by rapid Ca2 + -dependent endocytosis. J Cell Biol. 2008;180(5):905.

36. Johansson AC, Appelqvist H, Nilsson C, Kagedal K, Roberg K, Ollinger K. Regulation of apoptosis-associated lysosomal membrane permeabilization. Apoptosis. 2010;15(5):527-40.

37. Hernot S, Klibanov A. Microbubbles in ultrasound-triggered drug and gene delivery. Adv Drug Deliv Rev. 2008;60(10):1153-66.

38. D'Souza AL, Tseng JR, Pauly KB, Guccione S, Rosenberg J, Gambhir SS, Glazer GM. A strategy for blood biomarker amplification and localization using ultrasound. Proc Natl Acad Sci U S A. 2009;106(40):17152-7.

\section{Submit your next manuscript to BioMed Central and we will help you at every step:}

- We accept pre-submission inquiries

- Our selector tool helps you to find the most relevant journal

- We provide round the clock customer support

- Convenient online submission

- Thorough peer review

- Inclusion in PubMed and all major indexing services

- Maximum visibility for your research

Submit your manuscript at www.biomedcentral.com/submit 